\title{
EL RÍO ARLANZA EN CABECERA: EVOLUCIÓN RECIENTE Y TENDENCIAS EN CONDICIONES DE CAMBIO GLOBAL
}

\author{
J. MACHIN, A. NAVAS, S. DOMENECH y M. LOPEZ-VICENTE \\ Estación Experimental de Aula Dei, CSIC. Apartado 202, 50080 Zaragoza \\ Correo electrónico de contacto: jmac@eead.csic.es
}

\begin{abstract}
RESUMEN: Las cuencas de cabecera de montaña son áreas extremadamente sensibles a los procesos de cambio global. El impacto antrópico y el cambio climático van a afectar a los regímenes fluviales y modificar los recursos hídricos. Los pronósticos generales para la eco-región mediterránea coinciden en una reducción de precipitaciones acompañada de un incremento de temperaturas y una mayor frecuencia de eventos extremos tanto sequías como inundaciones. La cabecera de cuenca del Arlanza se ha seleccionado como sector de control de cambios para proceder a un análisis de la evolución reciente y tendencias de los aportes y caudales en la estación de aforo de Castrovido para el periodo hidrológico 1960-2002. Los resultados confirman la existencia de una tendencia general a la reducción de aportes, mas marcada a partir de 1980. Desde 1995 se producen las avenidas con los caudales mas extremos, así como el mayor rango de variación de caudales máximos anuales del periodo estudiado. En estas condiciones de cambio y para promover una planificación racional de los recursos hídricos, es de interés el seguimiento de tendencias y pautas de aportes y caudales en cuencas forestales de cabecera, ya que constituyen las principales fuentes suministradoras de agua.
\end{abstract}

SUMMARY: The headwater catchments in mountain areas are highly sensitive under conditions of global change and their fluvial regimes and hydrological resources are likely to be notably modified. The general predictions for the Mediterranean region are the increase in temperature and decrease in rainfall together with a higher frequency of extreme events, both droughts and floods. The Arlanza basin at the headwater was selected as a control sector to examine the recent evolution and trends in discharge. At the gauging station of Castrovido, a general decreasing trend of annual discharge was found for the 1960-2002 hydrological period. The main decrease period started in 1980 and discharge was reduced by almost 50\%. Since 1995, extreme floods were relatively more frequent and also the largest range of variation in the 
maxima annual flood was found. Under climatic change conditions and in order to promote the rational planning of hydrological resources, a fundamental understanding of patterns and trends of discharge is required but specially in forest catchments as their headwaters are main sources of water to the lowlands.

Palabras clave: recursos hídricos, evolución, tendencias, cambio climático, cabecera de cuenca, Arlanza, Cuenca del Duero, España.

Key words: hydrological resources, evolution, trends, climatic change, Arlanza, headwater catchment, Duero basin. Spain.

Enviado el 2 de noviembre de 2005 Aceptado el 12 de diciembre de 2005

\section{Introducción}

En el contexto climático y fisiográfico del territorio español, los recursos hídricos superficiales, su cuantificación, disponibilidad y uso plantean una cuestión medioambiental de primer orden. Existe actualmente un consenso científico generalizado sobre la existencia del cambio climático y en los últimos años son múltiples las investigaciones sobre la incidencia de sus efectos a escala planetaria (Easterling et al., 2000; Parry et al., 2000). En Europa, los países del área mediterránea son los que mas riesgo presentan de sufrir cambios ambientales que afectarán a los recursos de agua y suelo y que podrían conducir a la desertificación (Navas et al., 2005)

Nearing et al. (2004) indican que el calentamiento global conducirá a un ciclo hidrológico mas vigoroso con una mayor frecuencia de eventos de mayor intensidad pluviométrica. Ayala-Carcedo e Iglesias (2000) señalan que la variación de las pautas temporales de los aportes en diversas cuencas españolas medidos a través del cambio del coeficiente de variación interanual, sugiere un aumento de los eventos extremos tanto de inundaciones como de sequías. Estas pautas han sido también recientemente pronosticadas a nivel mundial por Meehl et al. (2005) que constatan un incremento de desastres naturales a lo largo del siglo XX.

Si bien la regulación de los recursos hídricos en España ha aumentado paulatinamente a lo largo del siglo XX, según Vicente Serrano (2004) en su estudio sobre la evolución espacio-temporal de las sequías, aún siguen existiendo problemas de gestión y planificación que conducen al desabastecimiento como el que se produjo en la sequía de 1995 y en la de este mismo año 2005, que no sólo ha afectado a las regiones semiáridas sino a amplios sectores de la geografía peninsular tradicionalmente considerados como la España húmeda. 
En la actualidad, el problema medioambiental que se plantea viene dado por la incertidumbre del agua disponible, lo que eventualmente condiciona la productividad de los sistemas agrarios e incluso el mantenimiento de la vegetación natural. La evaluación de los recursos hídricos superficiales y las previsiones sobre su posible evolución requieren como punto de partida el análisis de las series de caudales existentes con objeto de valorar su disponibilidad final para asegurar la sostenibilidad de los agroecosistemas. El análisis de la distribución de caudales y de sus cambios a lo largo del año proporciona criterios científicos de base necesarios para la planificación de los recursos hídricos. El seguimiento de sus pautas en el tiempo y en el espacio puede servir para identificar posibles cambios ambientales (García Ruiz et al., 2001).

Las cabeceras de cuencas forestales en zonas de montaña media-alta pueden ser indicadores de respuesta más rápida a los procesos de cambio global. Las condiciones de menor transformación que generalmente se suelen encontrar en ellas, las menores afecciones antrópicas en zonas de menor densidad de población y por consiguiente la relativamente menor explotación de los recursos hídricos hacen que estas cuencas de cabecera puedan servir como sectores de control de cambios ambientales y antrópicos para poder así analizar su impacto en ambientes de menor confluencia de factores.

En este marco de cambio global, cobra especial interés medioambiental el estudio de los regímenes fluviales en cuencas de montaña representativas, que albergan una gran biodiversidad y en las que la sostenibilidad de sus ecosistemas puede verse seriamente afectada. Dado el horizonte de incertidumbre de los recursos hídricos, su planificación y gestión sostenible requiere como tarea fundamental una primera fase de evaluación de los mismos y de su evolución temporal que permita confirmar o descartar posibles tendencias.

Este estudio se centra en el análisis de características de los recursos hídricos del río Arlanza en Castrovido. Esta parte de la cuenca en cabecera con densas masas forestales se ha seleccionado como representativa de una cuenca de montaña del Norte de España para realizar el seguimiento de las características de su régimen fluvial y como sector de control de posibles cambios en distintos periodos hidrológicos.

\section{Material y métodos.}

El río Arlanza nace en Fuente Sanza en las inmediaciones de Quintanar de la Sierra, al pie de la Sierra de Neila (2048 m) en el marco agreste de la Sierra de la Demanda (Figura 1). En este sector, el río drena la cabecera de cuenca constituida exclusivamente por materiales del Cretácico inferior de naturaleza detrítica, fundamentalmente conglomerados, gravas, arenas y arcillas. Rompiendo esta monotonía litoestratigráfica, en las inmediaciones de Palacios de la Sierra, afloran materiales del Cámbrico y del Jurásico inferior. En este recorrido aparecen los únicos depósitos cuaternarios de cierta entidad que constituyen el aluvial y la llanura de inundación del río. Pasado el paraje de Los Vados, el Arlanza discurre hasta Castrovido donde se sitúa la estación de aforo de 


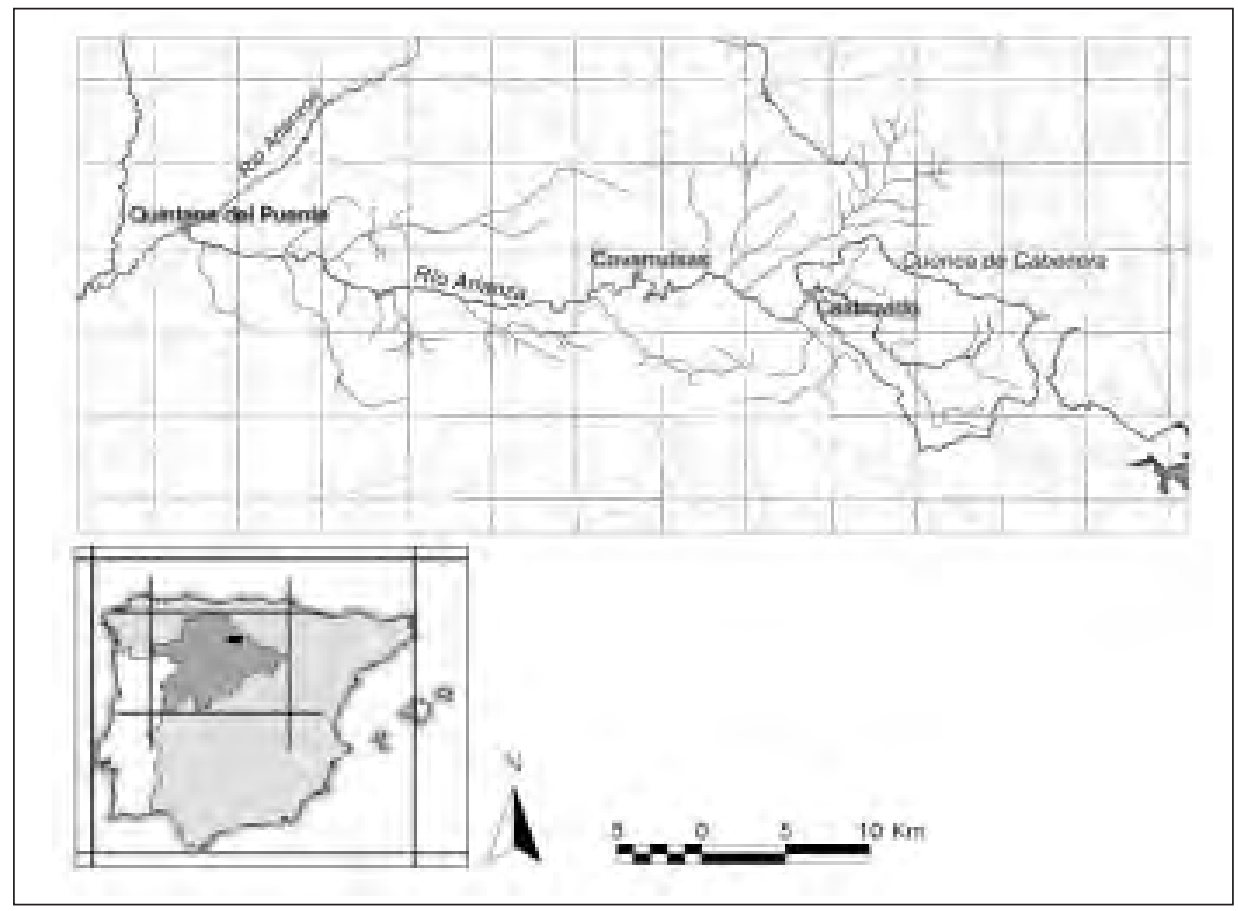

Figura 1. Situación geográfica del área de estudio.

la Confederación Hidrográfica del Duero (CHD). En este sector el río discurre encajado entre areniscas, conglomerados y margas del Cretácico inferior, salvando fuertes desniveles cuya altura sobre el cauce supera los $100 \mathrm{~m}$.

En el área, el clima es continental con inviernos muy rigurosos y veranos frescos. La precipitación media anual oscila entre 780 y mas de $1100 \mathrm{~mm}$ en zonas altas de la Sierra. La vegetación es muy diversa y se distribuye en función de las condiciones de precipitación y humedad. Por encima de los $1000 \mathrm{~m}$ aparecen densas masas forestales de pinos (Pinus nigra) que han predominado en el área durante todo el Holoceno (Franco Múgica et al., 2001). La explotación maderera del pinar es un importante recurso de la economía tradicional en la zona. En este sector de cabecera hay además una gran variedad de especies (acebo, avellano, tejo y enebro). Por debajo de los $1000 \mathrm{~m}$ predominan los robledales de Quercus pyrenaica. Existen diversas especies arbustivas (endrino, majuelo, zarzamoras) y matorrales (brezo, genista, jara, gayuba y enebro). En el curso del río se encuentra un denso bosque de ribera con saucedas y alisales.

Los datos de aportes y caudales se han extraído de las series "resumen de datos históricos" y de los aforos diarios de la Confederación Hidrográfica del Duero (CHD) en Castrovido para el periodo hidrológico comprendido entre 1960, en que se inicia la serie, hasta 2002. 


\section{Afecciones del cambio global a los recursos hídricos del Arlanza en cabecera.}

\subsection{Análisis de aportes}

El río Arlanza en Castrovido recoge los caudales de la cabecera de la cuenca. Con una superficie drenada de $349 \mathrm{~km}^{2}$, este tramo pertenece plenamente al área de montaña. Como corresponde a un río de régimen pluvio-nival de la montaña media-alta y clima continental, los mayores aportes y las avenidas se registran en los meses de invierno de diciembre a febrero, siendo enero el mes en el que los aportes medios son mas elevados $\left(24 \mathrm{Hm}^{3}\right)$. Marzo, abril, noviembre y mayo, son por este orden, meses de aportes medios aún elevados (entre 13 y $19 \mathrm{Hm}^{3}$ ). En junio se produce un marcado descenso (6.8 $\mathrm{Hm}^{3}$ ) para entrar claramente en el estiaje que se prolonga durante los meses de verano, siendo agosto y septiembre, los de menores aportes medios (1 y $1.4 \mathrm{Hm}^{3}$ ). El aporte mínimo $\left(0.01 \mathrm{Hm}^{3}\right)$ se registró en el mes de Septiembre de 1984. En octubre se obser-

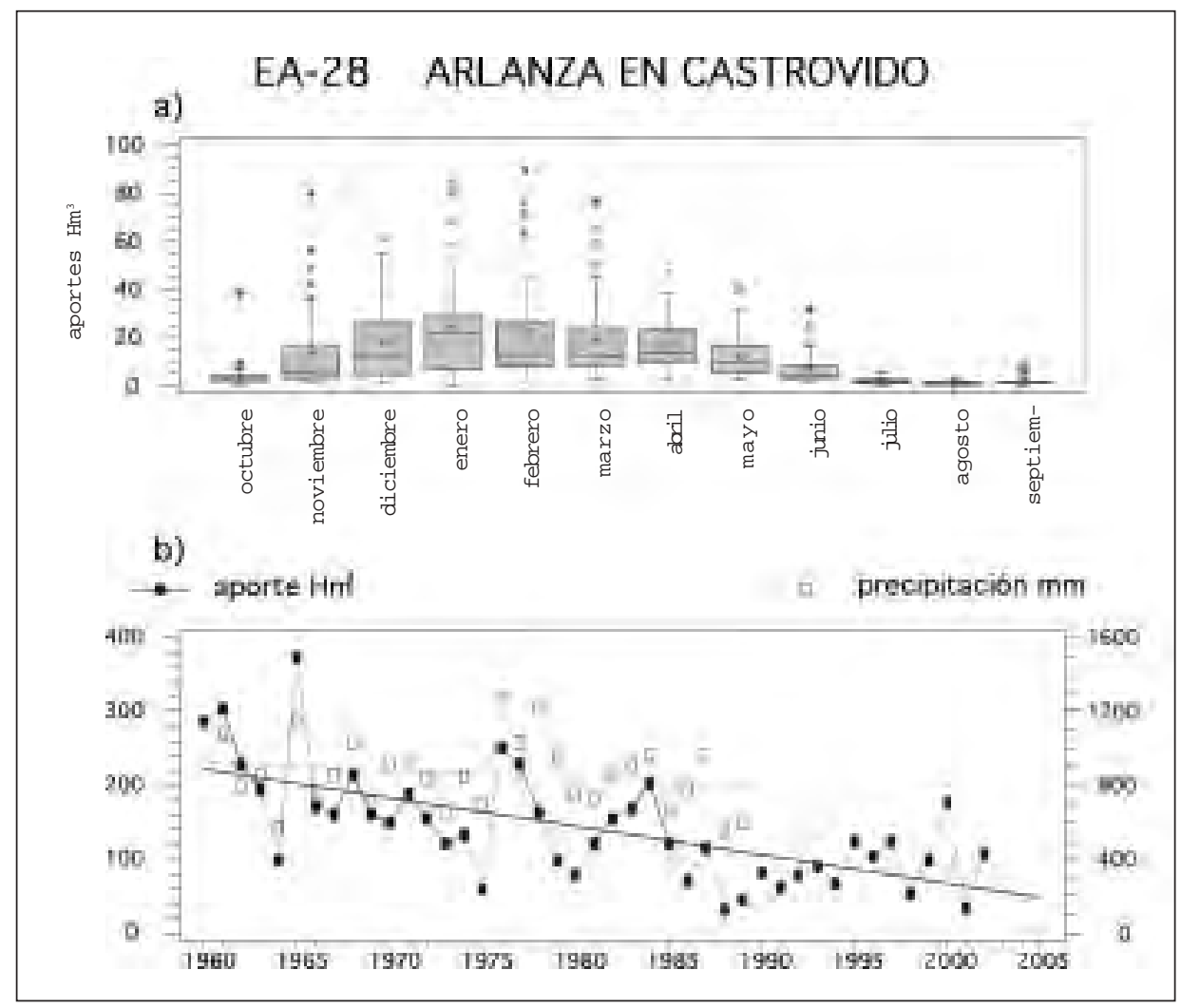

Figura 2. a) Valores medios, desviación estándar, mediana, valores de los percentiles 25 y 75 y valores extremos para los meses del año hidrológico (1960-2002).

b) variación del aporte anual y la precipitación y sus tendencias en el Arlanza en Castrovido para el periodo hidrológico 1960-2002. 
Tabla 1. Estadística básica de los aportes mensuales del Arlanza en Castrovido en el periodo hidrológico 1960-2002.

\begin{tabular}{|l|r|r|r|r|r|r|r|r|r|r|r|r|}
\hline $\begin{array}{l}\text { Arlanza en } \\
\text { Castrovido }\end{array}$ & Oct & Nov & Dic & Ene & Feb & Mar & Abr & May & Jun & Jul & Ago & Sep \\
\hline n & 43 & 43 & 43 & 43 & 43 & 43 & 43 & 43 & 43 & 43 & 43 & 43 \\
media & 4.1 & 13.3 & 18.3 & 23.7 & 21.8 & 19.2 & 16.7 & 12.2 & 6.8 & 2.1 & 1.0 & 1.4 \\
ds & 5.9 & 17.1 & 16.5 & 20.7 & 21.7 & 17.7 & 10.1 & 9.4 & 6.3 & 1.4 & 0.7 & 1.5 \\
mínimo & 0 & 0.6 & 0.9 & 0.4 & 1.6 & 1.9 & 2.7 & 2.0 & 1.2 & 0.2 & 0.1 & 0.0 \\
máximo & 38.6 & 79.6 & 61.2 & 84.3 & 90.2 & 76.9 & 46.4 & 41.9 & 31.6 & 5.3 & 3.0 & 8.0 \\
CV \% & 143.4 & 128.1 & 90.6 & 87.2 & 99.5 & 92.1 & 60.3 & 76.8 & 93.6 & 66.8 & 71.2 & 106.3 \\
\hline
\end{tabular}

Tabla 2. Estadística básica de los aportes medios anuales del Arlanza en Castrovido para el periodo hidrológico 1960-2002 y de precipitación en el periodo 1961-1990.

\begin{tabular}{|l|c|c|}
\hline $\begin{array}{l}\text { EA-28 } \\
\text { Arlanza en Castrovido }\end{array}$ & $\begin{array}{c}\text { aporte } \\
\mathbf{H m}^{3}\end{array}$ & $\begin{array}{c}\text { precipitación } \\
\text { mm }\end{array}$ \\
\hline $\mathrm{n}$ & 43 & 29 \\
media & 140.7 & 859.9 \\
desviación estándar & 75.1 & 185.1 \\
mínimo & 30.1 & 548.0 \\
máximo & 374.5 & 1269.0 \\
coeficiente de variación & 53.4 & 21.5 \\
\hline
\end{tabular}

va ya un cambio de tendencia al incremento y se llega a registrar algún aporte importante, lo que se refleja en el correspondiente valor del coeficiente de variación que es el más alto de los meses del año hidrológico en el periodo estudiado. La Figura 2a y la Tabla 1 muestran los valores medios de aportes, desviación estándar y otros parámetros aritméticos (mediana, valores de los percentiles 25 y 75 y valores extremos) para los meses del año hidrológico en el periodo 1960 a 2002.

Como se observa en la Figura 2b, existe una tendencia significativa hacia el descenso de los aportes anuales $(\mathrm{r}=-0.645)$. El aporte anual y la precipitación presentan un marcado paralelismo evidenciando la relación directa entre ambos $(r=0.723)$. En cuanto a la precipitación, si bien los registros no están completos para el periodo estudiado (datos en 29 de los 43 años hidrológicos), se observa una disminución en el tiempo, aunque esta tendencia no es estadísticamente significativa $(r=-0.187)$, lo que podría deberse al propio tamaño de la serie de datos. 
La Tabla 2 presenta el amplio rango de variación de los aportes anuales que oscilan de 30 a $375 \mathrm{Hm}^{3}$ para el periodo 1960-2002. Desde el año 1960 hasta 1980 se producen las mayores oscilaciones en los aportes. En estos 20 años, se registran los máximos anuales alcanzando en $1965 \operatorname{los} 375 \mathrm{Hm}^{3}$, mientras que sólo en tres años dentro de estas dos décadas se producen aportes anuales inferiores a $100 \mathrm{Hm}^{3}$. En marcado contraste, a partir de 1980, un total de 13 años registran aportes anuales inferiores a 100 $\mathrm{Hm}^{3}$.

Como se ha indicado, la tendencia significativa a la disminución de aportes con el tiempo, más marcada aún en las dos últimas décadas, podría deberse a un conjunto de factores entre los que cabría mencionar el mayor consumo de agua en la cuenca, los cambios de uso del suelo, así como la posible disminución de la precipitación. Esta tendencia coincide con la observada en algunas cabeceras de cuencas pirenaicas (ValeroGarcés et al., 1999, García et al., 2001). Asimismo, una reciente investigación en el valle del Ziz (límite con el Sahara marroquí) (Navas et al., 2005), ha puesto de manifiesto que la sequía desencadenada a partir de 1980 y que aún perdura, ha conducido a la drástica disminución de los recursos hídricos, a la degradación de los agroecosistemas por salinización y al abandono de tierras, amenazando la conservación del palmeral centenario de Talfilalet. La fecha de inicio de la sequía en 1980 que es también coincidente con la del descenso de aportes que registra el Arlanza en cabecera, podrían tener relación con el contexto general de cambio climático, salvando la distancia geográfica y de caracteres fisiográficos entre ambas regiones.

La evolución temporal de los aportes mensuales confirma también esta tendencia regresiva. Según se observa en la Tabla 3, existe una disminución de los aportes en todos los meses siendo, en general, más significativa en los de altos caudales. No obstante, también en el estiaje, la reducción de caudales produce una significativa disminución de los aportes. Estas tendencias podrían agudizarse con el pronóstico para las cuencas del Norte de España, de un aumento de la temperatura media anual de mas de $2^{\circ} \mathrm{C}$ y reducciones del $2 \%$ en la precipitación anual (Ayala-Carcedo e Iglesias, 2000).

Tabla 3. Coeficientes de correlación entre aporte medio mensual y tiempo del Arlanza en Castrovido para el periodo 1960-2002. (*** $p \searrow 0.01, * * p \searrow .05, * p \searrow .1$ )

\begin{tabular}{|c|c|c|c|c|c|c|c|c|c|c|c|}
\hline oct & nov & dic & ene & feb & mar & abr & may & jun & jul & ago & sep \\
\hline-0.242 & -0.469 & -0.191 & -0391 & -0.389 & -0.509 & -0.428 & -0.293 & -0.320 & -0.281 & -0260 & -0.368 \\
\hline$*$ & $* * *$ & $*$ & $* * *$ & $* * *$ & $* * *$ & $* * *$ & $*$ & $* *$ & $*$ & $*$ & $* *$ \\
\hline
\end{tabular}

La mayor significación que ya se observa en las tendencias regresivas de los aportes en marzo y abril podría acentuarse si como se espera para el Norte de España, se produce además una disminución de la precipitación nival por el aumento de la temperatura. Aumento que se estima podría alcanzar un $25 \%$ para el conjunto de España en 
2060 (Ayala-Carcedo y Piserra, 2000).

Una investigación reciente (Schröter et al., 2005) examina para Europa diferentes escenarios que se verán afectados por el cambio climático. Entre los principales impactos que se mencionan para nuestra eco-región destacan los riesgos por la pérdida de fertilidad del suelo y la disminución de agua útil que afectará especialmente a regiones del Mediterráneo y de montaña. En este trabajo las cuencas de montaña se señalan como "áreas desproporcionadamente sensibles al cambio climático" en las que el desplazamiento de las nieves a mayores cotas perturbará los regímenes fluviales.

\subsection{Análisis de caudales}

El caudal medio del Arlanza en Castrovido para el período hidrológico estudiado es de $4.52 \mathrm{~m}^{3} / \mathrm{s}$, valor relativamente bajo por ser una estación de cabecera. Se observa una tendencia rítmica de aguas altas todos los años en invierno, como le correspondería a un típico río de régimen pluvial oceánico. De esta misma forma, el río registra sus caudales mínimos en la época estival, momento de sus aguas más bajas, quedando prácticamente seco en determinadas fechas (a veces se han llegado a registrar tan sólo 0.001 $\mathrm{m}^{3} / \mathrm{s}$ ). La variabilidad del caudal del río es alta, registrándose valores que comprenden desde los $172 \mathrm{~m}^{3} / \mathrm{s}$ hasta la práctica inexistencia de caudal en épocas de mayor estiaje. Asimismo, la variación de los caudales máximos y máximos instantáneos es amplia, desde valores ciertamente bajos $\left(4 \mathrm{~m}^{3} / \mathrm{s}\right.$ ) a máximos extremos de 172 y $304 \mathrm{~m}^{3} / \mathrm{s}$ respec-

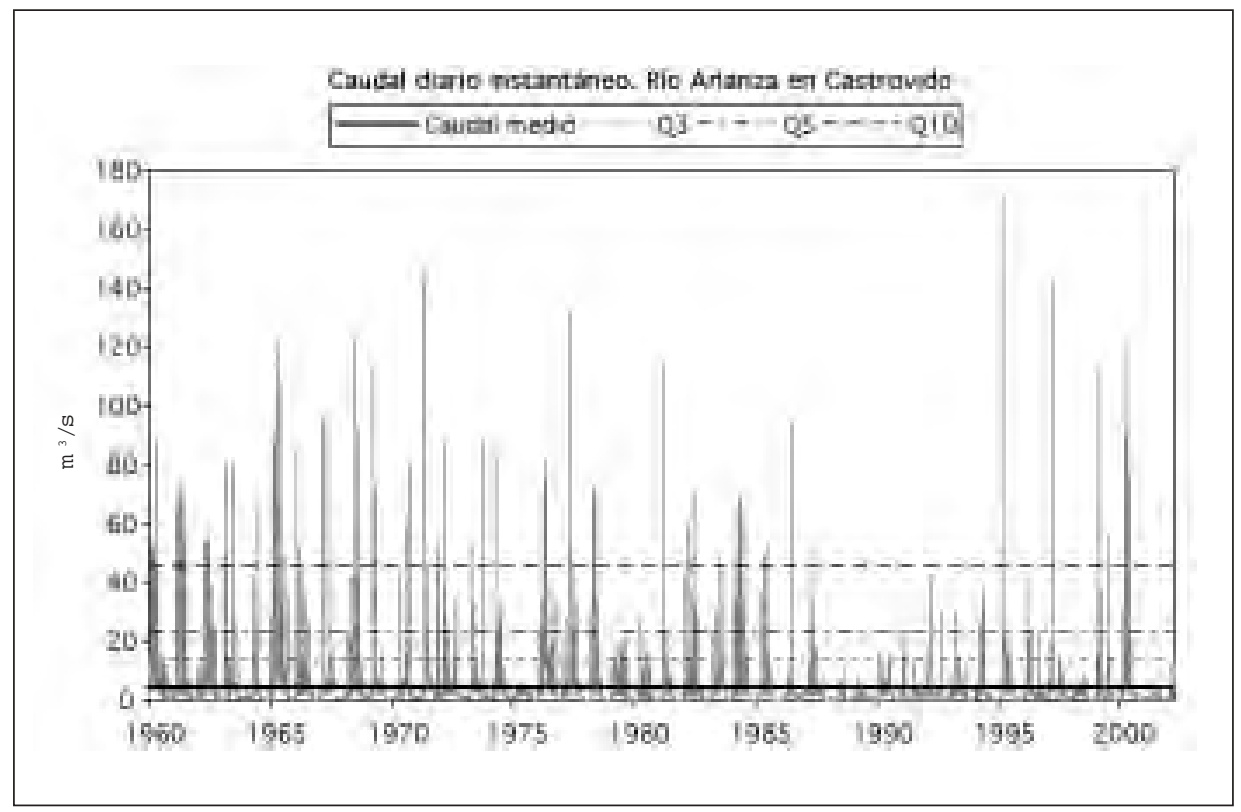

Figura 3. Distribución de caudales diarios instantáneos del río Arlanza en Castrovido y los correspondientes a 3, 5 y 10 veces el caudal medio anual en el periodo hidrológico 1960-2002. 
tivamente.

Tal y como ilustra la Figura 3, la distribución de los caudales en el período analizado no es homogénea. Bien es cierto que las aguas tienen una distribución anual similar, pero la tendencia en las últimas décadas refleja una realidad distinta, y es que los episodios de aguas altas tienen mayor magnitud que en épocas pasadas, por lo que el carácter extremo de las avenidas se acentúa más desde los noventa. Desde 1982 hasta los noventa, los episodios de crecidas registraban caudales cada vez menores, hasta que en fechas recientes, la tendencia se invierte de modo que ahora el río Arlanza registra crecidas de mayor entidad.

Cabe destacar también el hecho que desde 1988 hasta 1996, los caudales registrados en Castrovido fueron muy inferiores a los de épocas anteriores mostrando una tendencia regresiva. En principio, cabe pensar que la disminución de los caudales estaría relacionada con la de las precipitaciones. No obstante, para confirmarlo se requiere un registro mas amplio ya que éste se interrumpe en 1990. Otras causas conducentes a la disminución de caudales podrían ser el incremento de la cubierta forestal en la cuenca que reduciría la escorrentía (Beguería et al., 2003), además del mayor gasto de agua por parte de los usuarios.

Como indicadores de variaciones de caudal en el régimen fluvial de un río se puede analizar la frecuencia de días en los que se supera un determinado umbral. Estos umbrales son de utilidad para definir a un evento como avenida y han sido utilizados por diversos autores (García Ruiz et al., 2001, López Moreno et al., 2003).

El coeficiente Qx3 (Q3) expresa el valor alcanzado cuando el río registra el triple de su caudal medio. En Castrovido, aparecen 1086 episodios que superan este umbral, es decir, un $7 \%$ de los datos de caudal son aguas altas. Los eventos que superan por cinco el caudal del Arlanza a su paso por Castrovido (559) representan menos del $4 \%$. Mientras que las crecidas de mayor magnitud, representadas al multiplicar por diez el caudal medio del río, suponen el $1 \%$ de los eventos. El caudal máximo alcanzado en la serie de tiempo $\left(172 \mathrm{~m}^{3} / \mathrm{s}\right)$, equivaldría a una crecida de orden 38 (Q38).

\subsubsection{El comportamiento del régimen mensual del Arlanza en cabecera}

Para caracterizar el régimen fluvial del Arlanza en Castrovido se analiza la variación media de su caudal a lo largo del año. El uso de los caudales relativos, obtenidos de la división del caudal medio mensual entre el módulo o caudal medio anual permite establecer una categoría de valores que oscilan de cero a tres y comparar espacial y temporalmente los caudales (Tabla 4).

Como se puede ver en la Figura 4, el comportamiento estacional del Arlanza es diferente en función del mes del que se trate. Así, el río posee sus momentos de aguas más altas en los meses de Enero y Febrero. Este máximo se debe fundamentalmente a 
Tabla 4. Caudales mensuales relativos del río Arlanza en Castrovido.

\begin{tabular}{|l|c|c|c|c|c|c|c|c|c|c|c|c|}
\hline & Oct & Nov & Dic & Ene & Feb & Mar & Abr & May & Jun & Jul & Ago & Sep \\
\hline $\begin{array}{l}\text { Caudal medio } \\
\text { mensual } \mathbf{~ m}^{3} / \mathbf{s}\end{array}$ & 1.6 & 5.1 & 6.8 & 8.8 & 9.0 & 7.2 & 6.5 & 4.6 & 2.7 & 0.8 & 0.4 & 0.6 \\
Caudal relativo & 0.35 & 1.14 & 1.51 & 1.94 & 1.99 & 1.60 & 1.45 & 1.02 & 0.59 & 0.18 & 0.09 & 0.13 \\
\hline
\end{tabular}

precipitaciones líquidas de carácter frontal, asociadas al paso de frentes fríos (desde el Oeste) propias de nuestras latitudes. Se observa también un máximo secundario en el mes de Abril. Este ligero aumento de caudal se explica gracias a las precipitaciones en forma de nieve caídas meses atrás y cuya fusión tras las primeras lluvias de primavera incrementan el caudal del río. La alimentación en cabecera del Arlanza no es nival pura, por lo que no experimenta la retención típica de nieve en los meses invernales y el consiguiente descenso de caudales, pero sí que registra un período de aguas altas ligadas a esta fusión nival en el mes de Abril debido a las primeras lluvias primaverales. La tendencia regresiva de los aportes en los meses de Marzo y Abril, la mas significativa de todos los meses del año, podría ser un indicio del desplazamiento de la nieve a cotas altitudinales mas elevadas (Schröter et al., 2005), por lo que no se puede descartar una progresiva disminución de aportes procedentes de la fusión nival.

El caudal del Arlanza desciende hasta que en el verano se registran los meses de

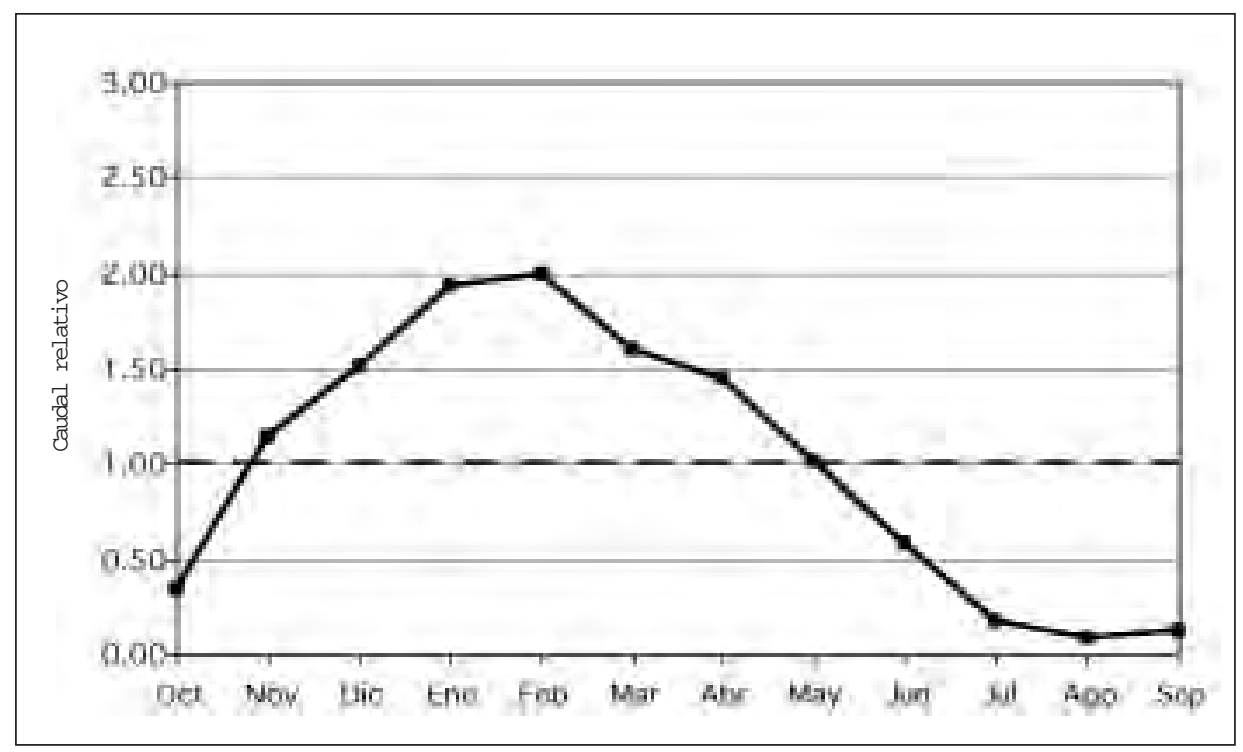

Figura 4. Caudal mensual relativo del río Arlanza en Castrovido. 
aguas bajas. Desde Junio hasta Octubre, el río posee caudales inferiores al módulo (caudal medio anual) encontrando un período de aguas muy bajas en el mes de Agosto (mínimo anual). La escasez de precipitaciones del ámbito mediterráneo es la razón que explica este comportamiento de los caudales. El régimen pluviométrico de nuestras latitudes imprime este descenso de caudal tan brusco. Como se ha indicado, las tendencias regresivas en los aportes del Arlanza en Castrovido son generales para estos meses de aguas bajas. Será necesario contrastar si se consolida esta tendencia para periodos futuros.

\subsubsection{Análisis de avenidas}

Además de la incertidumbre por la disponibilidad de recursos hídricos y en contraposición a la escasez de aportes durante el estiaje otras cuestiones preocupantes a nivel medioambiental y por las graves afecciones para las poblaciones ribereñas es el régimen de avenidas. En la Figura 5 se presentan los caudales máximos y máximos instantáneos anuales del periodo estudiado. Se puede observar que de 1960 a 1980, un total de 16 años tuvieron caudales máximos por debajo de $100 \mathrm{~m} / \mathrm{s}$, siendo los valores más frecuentes en torno a 60-80 m³ $\mathrm{m}^{3} / \mathrm{s}$. En las dos últimas décadas a partir de 1980, existe un predominio de caudales máximos con valores por debajo de $50 \mathrm{~m}^{3} / \mathrm{s}$ en 12 años. Sin embargo entre 1995 y 2000 se registran los caudales máximos y máximos instantáneos más extremos de todo el período hidrológico, alcanzando $172 \mathrm{~m}^{3} / \mathrm{s}$ y $230 \mathrm{~m}^{3} / \mathrm{s}$ respectivamente en el año 1995. Estos valores contrastan fuertemente con los datos del aporte anual ya que si bien la tendencia estadísticamente significativa es a la disminución de aportes, se observa en contraposi-

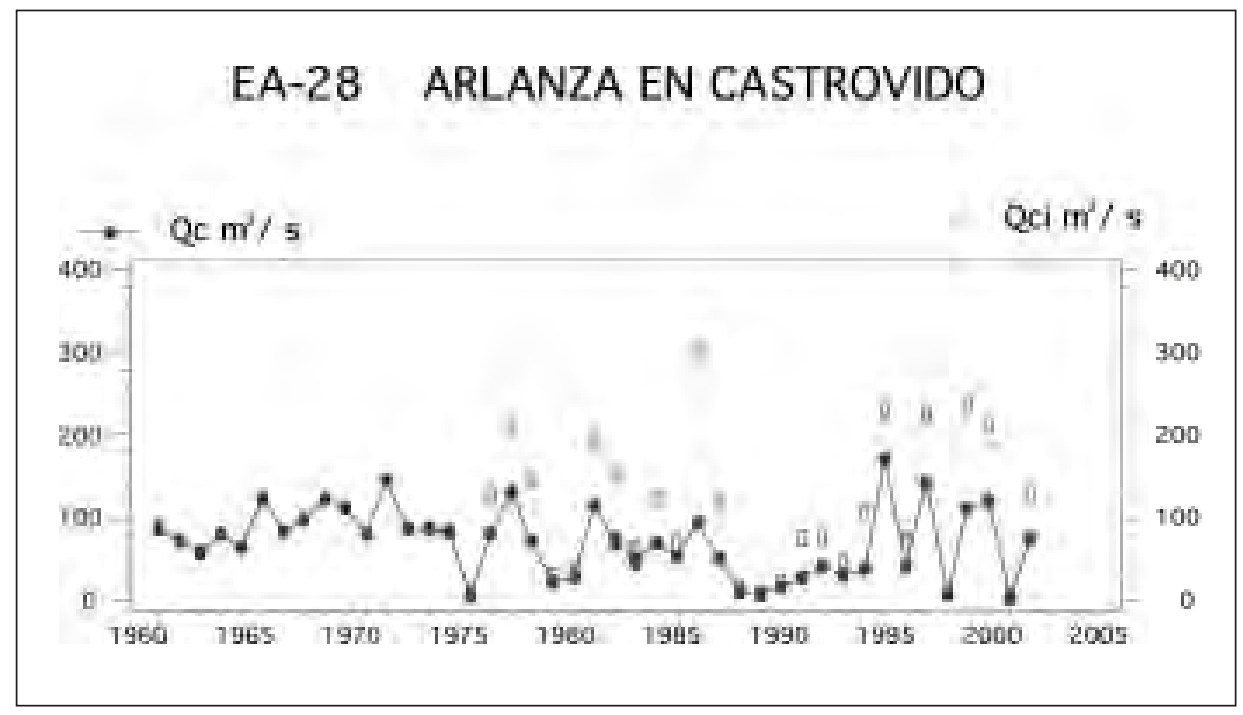

Figura 5. Caudales máximos y máximos instantáneos anuales del Arlanza en Castrovido en el periodo hidrológico 1960-2002. 
ción que entre 1995 y 2000, hay un marcado aumento de los caudales en las avenidas.

Estas pautas de aportes parecen estar relacionadas con el cambio climático, ya que en países del área mediterránea coexistirán situaciones de mayor sequía junto con eventos extremos causantes de avenidas. Considerando una escala temporal mas amplia, estudios sobre cambios climáticos durante el Holoceno en el área mediterránea realizados por Issar y Makover-Levin (1993) indican que los periodos mas cálidos de los últimos 10000 años han sido a su vez más áridos. Barriendos (1997) y Pfister et al. (1998) señalan que durante la Pequeña Edad del Hielo, entre los siglos XIV al XIX, se dieron en la península condiciones de mayor sequía con lluvias extremas ocasionales.

Otro análisis que se desprende de la variabilidad estacional de caudal es el de las crecidas del río para cada mes del año. El análisis de la distribución espacio temporal de las avenidas y de sus tendencias es necesario para conocer mejor el comportamiento hidrológico de los ríos, y muy especialmente en aquellas cuencas de marcada torrencialidad como la del río Arlanza. Si bien las crecidas están directamente relacionadas con la intensidad y cantidad de las precipitaciones, existen otros factores fisiográficos tales como la pendiente, litología y usos del suelo que también juegan un importante papel en su desencadenamiento y evolución.

La Figura 6 recoge el número de días por mes en los que el caudal del río alcanza el coeficiente Q3, Q5 y Q10. Así, se observa como los períodos de aguas altas (caracterizadas por el caudal Q3) se dan en el mes de Enero, con casi 8 días al mes, mientras que

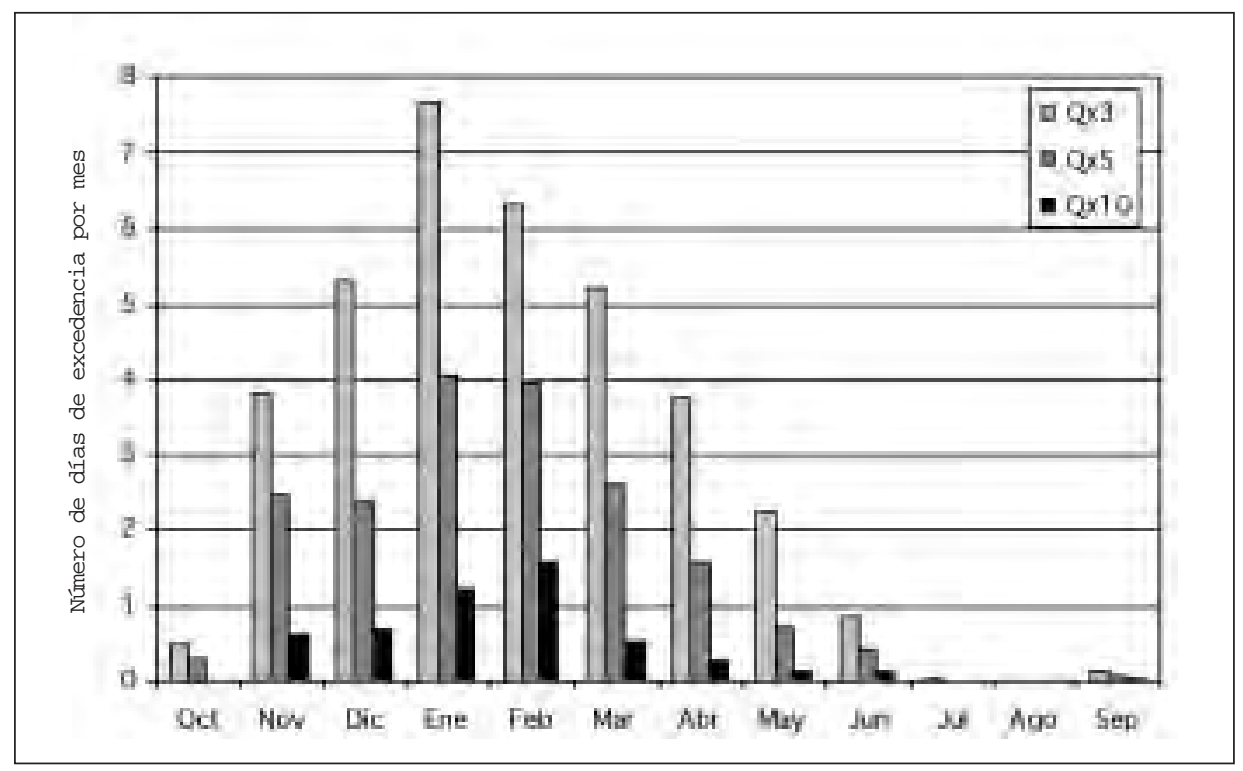

Figura 6. Número de días por mes en los que el caudal del Arlanza en Castrovido supera en 3, 5 y 10 la media anual. 
el régimen fluvial desvelaba que el mes de mayores caudales era el de Febrero. Las crecidas que multiplican por cinco el caudal medio anual (Q5) se producen en mayoría en los meses de Enero y Febrero (con cuatro días al mes en cada caso), mientras que las crecidas de mayor envergadura, las que suponen un aumento de diez veces el caudal medio (Q10) se registran en el mes de Febrero. Es decir, es este mes el que registra los episodios de mayor intensidad. Estas crecidas se deben fundamentalmente a las precipitaciones líquidas invernales, mientras que la fusión nival explica los episodios de aguas altas a finales de invierno y comienzos de la primavera (Marzo-Abril).

El cálculo de la probabilidad de ocurrencia de eventos extremos mediante métodos paramétricos se basa en el ajuste de determinadas distribuciones de probabilidad a las series de datos. Estas distribuciones son funciones matemáticas que relacionan la magnitud de un evento con su probabilidad de ocurrencia (Beguería, 2002). Los caudales esperados para diferentes períodos de retorno según el ajuste de Gumbel (1934), indican que un aumento tan súbito de caudal del Arlanza en Castrovido como el de la mayor avenida del periodo hidrológico estudiado, que es la de $172 \mathrm{~m}^{3} / \mathrm{s}$, se podrá dar con una frecuencia no superior a 26 años. El período de retorno calculado para una crecida de 72 $\mathrm{m}^{3} / \mathrm{s}$ (valor medio de los caudales máximos históricos) es tan sólo de dos años.

\subsubsection{Análisis de aguas bajas}

El Arlanza presenta un periodo de aguas bajas desde Junio hasta Octubre, en el cual los caudales son inferiores al módulo, alcanzándose una mayor frecuencia de valores mínimos en Agosto. Un análisis similar al realizado para las avenidas se aplica para clasificar los caudales de aguas bajas. Dividiendo el caudal medio por 3, 5 y 10 se obtienen los umbrales de $\mathrm{Q} / 3=0.905-1.507 \mathrm{~m}^{3} / \mathrm{s}, \mathrm{Q} / 5=0.451-0.904 \mathrm{~m}^{3} / \mathrm{s}$ y $\mathrm{Q} / 10=0.000$ a $0.450 \mathrm{~m}^{3} / \mathrm{s}$, que sirven para caracterizar el régimen de aguas bajas del río.

A diferencia de lo que ocurre en ríos de régimen nival en los que se pueden diferenciar aguas bajas de invierno o época fría de las de verano o época cálida (García et al., 1985), el régimen pluvial oceánico del Arlanza determina que las aguas bajas correspondan mayoritariamente al estiaje, con escasa presencia de valores mínimos de caudal en los meses de invierno.

Existe un claro predominio de aguas bajas ya que un $46 \%$ de los caudales diarios del periodo hidrológico estudiado están por debajo de $1.5 \mathrm{~m}^{3} / \mathrm{s}$. El mayor porcentaje (18 $\%$ ), corresponde a los caudales inferiores a $0.45 \mathrm{~m}^{3} / \mathrm{s}$. Como se puede observar en la Figura 7, para este intervalo, la mayor frecuencia se registra en el mes de Agosto. Para caudales inferiores a $0.904 \mathrm{~m}^{3} / \mathrm{s}$ se modifican las pautas ligeramente y los mayores porcentajes se reparten de una manera mas igualitaria de Julio a Octubre. La distribución de frecuencias para los caudales inferiores a $1.5 \mathrm{~m}^{3} / \mathrm{s}$ concentra los mayores porcentajes en Junio, y como cabría esperar una menor frecuencia en los meses de Julio y Agosto, ya que en estos meses predominan los caudales mínimos.

Dadas las características de fuerte estiaje que presenta el río y por su coincidencia 


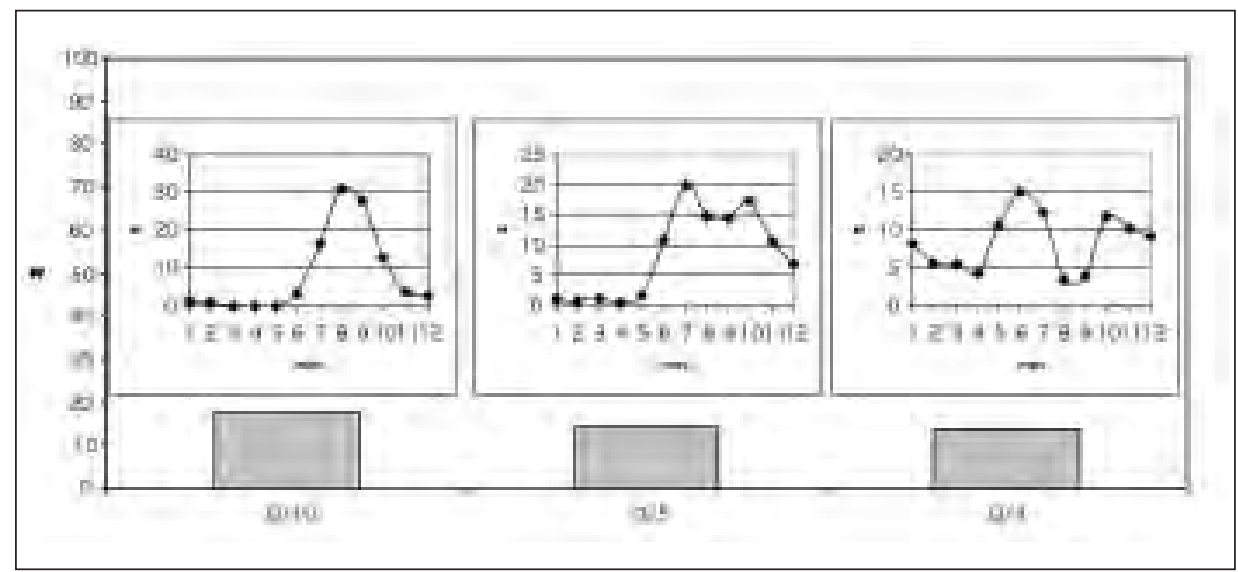

Figura 7. Porcentaje de días en los que el caudal del Arlanza en Castrovido es inferior en 3, 5 y 10 veces al medio anual y frecuencia de ocurrencia de los rangos de caudal Q/10 (0.000 a $\left.0.450 \mathrm{~m}^{3} / \mathrm{s}\right), Q / 5\left(0.451-0.904 \mathrm{~m}^{3} / \mathrm{s}\right)$ y $Q / 3\left(0.905-1.507 \mathrm{~m}^{3} / \mathrm{s}\right)$ en los meses del año.

con la época de mayor demanda de agua para cultivos y turismo, es de suponer que el efecto del cambio en el régimen climático agravará la situación, ya que el aumento de temperatura previsto conllevará un incremento de la evapotranspiración de la vegetación natural y de los cultivos, que a su vez requerirán mas agua. Este aumento de la temperatura se sumará a la reducción de aportes que algunos cálculos para la cuenca del Ebro estiman entre el 20 y 40\% (MIMAM, 1997; Ayala-Carcedo, 1996).

Los cambios climáticos que se pronostican se van a producir de una forma rápida, de modo que el seguimiento de sus pautas se hace aún mas necesario para una planificación adecuada de los recursos hídricos. En este sentido, se dispone de abundantes registros históricos en los que estudios paleoambientales han evidenciado la decadencia de poblaciones, causada por cambios mas o menos bruscos de las condiciones climáticas que condujeron a la escasez de agua (Messerli et al., 1993).

\subsection{Distribución del caudal por cuantiles.}

La distribución de los caudales del Arlanza en Castrovido según su aportación al régimen fluvial se analiza mediante la ordenación del caudal por cuantiles (López Moreno, 2005). Esta ordenación informa sobre si unos pocos días suelen llevar cantidades elevadas de caudal o si por el contrario, el régimen del río responde a bajos caudales pero durante más días del año. La ordenación de los caudales de menor a mayor y su agrupación en diez intervalos con igual número de datos de caudal se presenta en la Figura 8 a. Obtenidos los umbrales de los cuantiles de los caudales en la estación de aforo de Castrovido se determina la distribución de caudales. Como se puede observar en la Figura 8 b, la aportación de los caudales más altos al régimen del río (C10: 10.2 a $172 \mathrm{~m} 3 / \mathrm{s}$ ), supone más del $50 \%$ del caudal recogido en la serie de datos histórica. A gran 

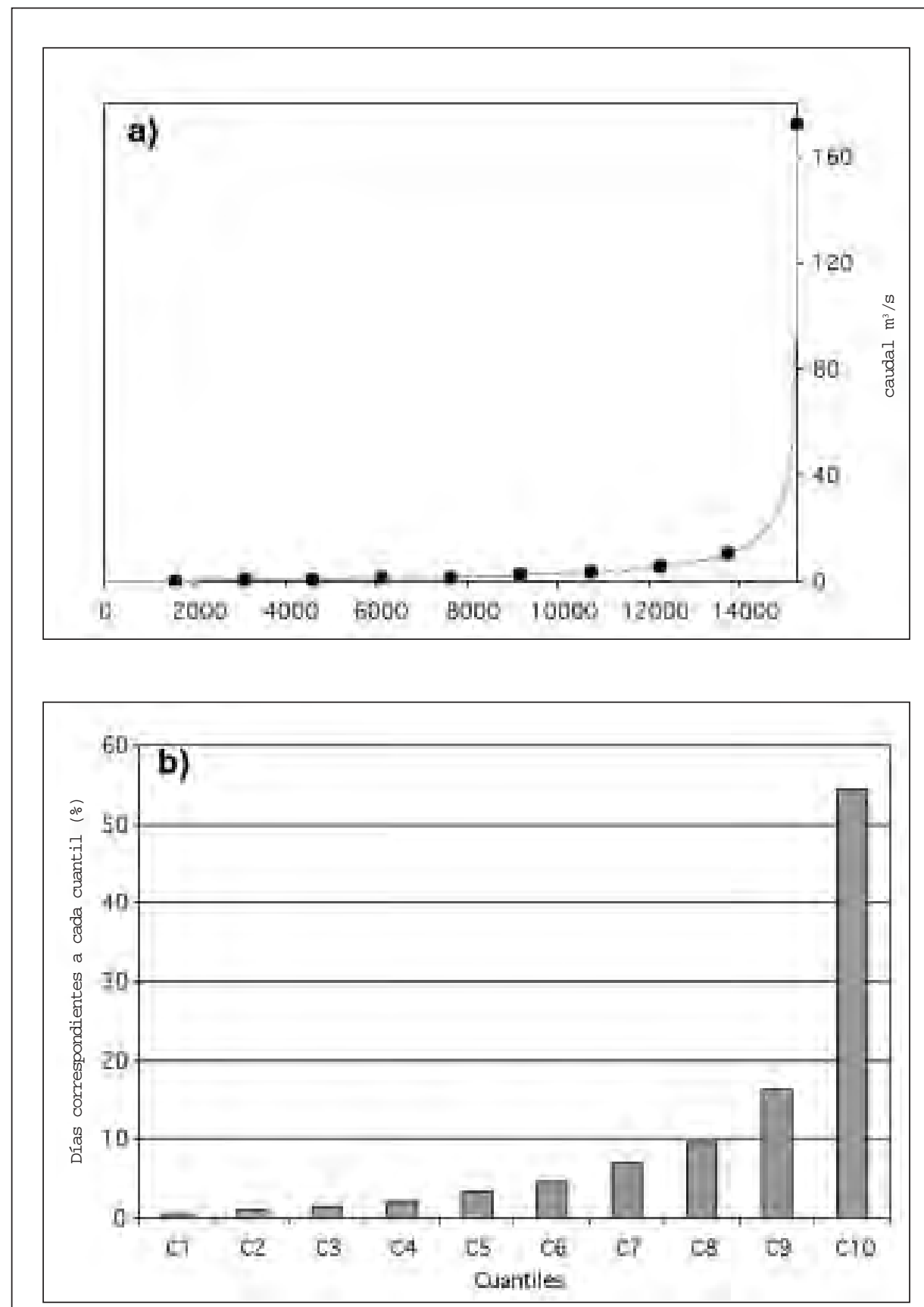

Figura 8. a) Cuantiles de caudal ordenados; b) aportaciones de caudal según cuantiles del Arlanza en Castrovido en el periodo hidrológico 1960-2002. 
distancia se sitúan los valores del noveno cuantil, entre 5.45 y $10.2 \mathrm{~m}^{3} / \mathrm{s}$, que suponen algo más del $15 \%$ de los datos de caudal. El resto de aportaciones poseen unos valores bajos en comparación con los datos de caudal de los dos últimos cuantiles. Se puede decir, por tanto, que el comportamiento del caudal del río Arlanza a su paso por Castrovido refleja pautas dispares en cuanto a los niveles de caudal registrados, encontrando bajos caudales en la mayor parte de los datos, suponiendo porcentajes de aportación pequeños, mientras que los episodios de aguas más altas suponen las mayores aportaciones con casi el $60 \%$ del caudal total del río.

\section{Conclusiones}

El río Arlanza tiene un régimen pluvial oceánico que se caracteriza por mantener caudales elevados de Noviembre a Mayo, es decir, el máximo es invernal, mientras que el estiaje es muy marcado. Un $46 \%$ de los registros de la serie corresponden a aguas bajas, con un predominio de caudales $(18 \%)$ inferiores a $0.45 \mathrm{~m}^{3} / \mathrm{s}$. Otra característica del Arlanza en cabecera son las crecidas de gran entidad, se trata de un río en el que los eventos extraordinarios son de gran importancia. Estas pautas se acentuarán con las previsiones de eventos mas extremos de sequía y precipitaciones.

En el periodo hidrológico estudiado se han observado fluctuaciones importantes de aportes y caudales. Se observa una diferencia clara en la serie con un periodo de mayores aportes entre 1960 y 1979, en el que el valor medio es de $186 \mathrm{Hm}^{3}$. A partir de 1980 se produce una importante reducción de hasta el $47 \%$, con un aporte medio de $100 \mathrm{Hm}^{3}$ entre 1980 y 2002. Desde 1995 se registra el mayor rango de variación de caudales máximos en todo el periodo hidrológico debido a eventos mas extremos, altos por fuertes avenidas y bajos por sequías.

Los aportes mensuales del Arlanza en cabecera muestran una tendencia regresiva significativa principalmente en los meses de aguas altas en invierno, pero aún es mas marcada y significativa la reducción de aportes en los meses de primavera, especialmente marzo y abril. Igualmente, los aportes tienden al descenso en los meses de verano.

La tendencia a la reducción de aportes, podría deberse a varias causas, en primer lugar a una disminución de las precipitaciones dada la relación directa que existe entre ambos parámetros. Pero también podrían contribuir a esta disminución un aumento de la superficie vegetal arbórea y arbustiva en la cuenca por cambios de usos del suelo, así como un mayor consumo de agua para diversos usos. Los resultados obtenidos pueden considerarse como buenos indicadores de las principales tendencias. No obstante, para analizar en profundidad la reducción general de aportes y su diferente significación según los meses del año hidrológico, es necesario realizar estudios posteriores de detalle que permitan determinar la incidencia relativa de cada uno de estos factores.

Las cabeceras de cuenca constituyen las principales áreas suministradoras de agua, por ello, el estudio de la evolución y tendencias de aportes y caudales son de interés para 
la planificación de los recursos hídricos. La cabecera del Arlanza se comporta como una zona ciertamente sensible en la que se aprecian rasgos relacionados con procesos de cambio global y puede servir, por tanto, para el control de cambios. Así, la tendencia regresiva de aportes, especialmente significativa en marzo y abril, podría estar relacionada con la disminución nival por su desplazamiento a mayores alturas. Además, las mayores fluctuaciones de caudales máximos anuales por inundaciones y sequías observada en los últimos años del periodo hidrológico estudiado, podrían tener relación con la mayor frecuencia de eventos extremos prevista para la región mediterránea.

\section{Agradecimientos}

Confederación Hidrográfica del Duero ha facilitado las series de datos de aforos y el resumen de datos histórico.

\section{Referencias bibliográficas}

AyAla-CARCEDO, F.J. (1996). Reducción de los recursos hídricos en España por el posible Cambio Climático. Tecnoambiente, 64: 43-48. Madrid.

Ayala-CARCEDo, F.J. y Iglesias, A. (2000). Impactos del posible Cambio Climático sobre los recursos hídricos, el diseño y la planificación hidrológica en la España Peninsular. En: El Cambio Climático. (Balairón, ed.). El Campo de las Ciencias y las Artes, Servicio de Estudios del BBVA, pp. 201-222, Madrid.

AYALA-CARCEDO, F.J. (2002). Notas sobre impactos físicos previsibles del cambio climático sobre los lagos y humedales españoles. En: III Congreso Ibérico de Planificación y Gestión de Aguas. (del Moral ed.) Fundación Nueva Cultura del Agua, pp. 360-364, Sevilla.

Ayala-CARCEDO, F.J. y PISERRA, MaT. (2000). Impactos del Cambio Climático sobre la economía y los seguros en Europa. En: Gestión de Riesgos y Seguros, MAFRE, pp. 15-20, Madrid

BARRIEndos, M. (1997). Climatic variations in the Iberian Peninsula during the Late Maunder minimum (Ad 1675-1715): an analyses of data from Rogation Ceremonies. The Holocene 7: 105-111.

BEGUERÍA, S. (2002). Revisión de métodos paramétricos para la estimación de la probabilidad de ocurrencia de eventos extremos en Climatología e Hidrología: el uso de series de excedencias y su comparación con las series de máximos anuales. En: La información climática como herramienta de gestión ambiental. VII Reunión Nacional de climatología, (Cuadrat, J.M., Vicente, S.M. y Saz, M.A., Ed.). pp. 83- 
92, Universidad de Zaragoza.

Beguería, S., López Moreno, J.I., Lorente, A., Seeger, M. y García-Ruiz, J.M. (2003). Assessing the effect of climate oscillations and land-use changes on streamflow in the Central Spanish Pyrenees. Ambio, 32(4): 283-286.

Easterling, D.R., Meehl, G.A., Parmesan, C., Changnon, S.A., Karl, T.R. y MEARnS, L.O. (2000). Climate Extremes: Observations, Modelling and Impacts, Science, 289: 2068-2074.

García-Ruiz, J.M., Puigdefabregas, J. y Creus, J. (1985). Los recursos hídricos superficiales del Alto Aragón. En: Colección de Estudios Altoaragoneses, 2. Instituto de Estudios Altoaragoneses, CSIC. Excma. Diputación Provincial de Huesca, 224 pág. Huesca.

García-Ruiz, J.M., Beguería Portugués, S., LóPeZ Moreno J.I., LORENTE GRIMA, A. y SEEgER, M. (2001). Los recursos hídricos superficiales del Pirineo aragonés y su evolución reciente. Geoforma ediciones S.L. 192 pág. Logroño.

Gumbel, E. J. (1934). Les valeurs extrêmes des distributions statistiques. Annales de l’I.H. Poincaré, 308 pág.

Issar, A. A. y Makover-Levin, D. (1993). Climate changes during the Holocene in the Mediterranean Region, en Angelakis and Issar, Diachronic Climate Impacts on Water Resources, NATO ASI Series, 16: 55-75.

LÓPEZ MoReno, J.I. (2005). Disponibilidad de recursos hídricos y gestión de los embalses en el Pirineo central español: interacciones entre variabilidad espacio-temporal de los recursos y el uso del agua bajo condiciones de cambio ambiental. Tesis Doctoral. Universidad de Zaragoza. Departamento de Geografía y Ordenación del Territorio. $491 \mathrm{pp}$.

López Moreno, J.I., Beguería, S. y García-Ruiz, J.M. (2003). Efecto de los embalses en el control de avenidas del Pirineo central español. Geographicalia, 44: 57-74.

Miмам (1998): Libro Blanco del agua, 855 pág. Madrid.

Meehl, G.A., Washington, W.M., Collins, W.D., Arblaster, J.M., Hu, A., Buja, L.E., Strand, W.G. y TENG, H. (2005). How much more global warming and sea level rise. Science, 307: 1769-1772.

Messerli, B., Grosjean, M., Bonani, G., Bürgi, A., Geyh, M., Graf, K., RAmseyer, K., Romero, H., Schotterer, U., Schreier H. y Vuille M. (1993). Climate change and natural resource dynamics of the Atacama Altiplano during the last 18,000 years: a preliminar synthesis. Mountain Research and Development, 13: 117127.

Franco Múgica F., García Antón M., Maldonado Ruiz J., Morla Juaristi C. y 
SaInZ Ollero H. (2001). The Holocene history of Pinus forests in the Spanish Northern Meseta. The Holocene, 11: 343-358.

Navas, A., Walling, D., Quine, T., Machín, J. y Soto, J. (2005). Soil redistribution patterns and factors along a transect in central Ebro basin (NE Spain) and its controls. En: Sediment Budgets 1 (Walling, D.E. \& Horowitz, A.J. Eds.) IAHS Publication, 291: 70-77.

Navas, A., Machín, J., Faleh, A. y SAdiki, A. (2005). Análisis de procesos de desertificación en el sur de Marruecos. Comparativa con áreas del valle Ebro y repercusiones socioeconómicas. Proyecto de cooperación hispano-marroquí. CSICUniversidad de Fez. Memoria proyecto.

NeARING, M.A., Pruski, F.F. y O’NEAL, M.R. (2004) Expected climate change impacts on soil erosion rates: a review. Journal of Soil and Water Conservation. 59: 43-50.

PARry, M., PARry, C. y M. LiVermore Eds. (2000). Valoración de los efectos potenciales del Cambio Climático en Europa (Informe ACACIA de la Comisión europea, Resumen y Conclusiones) Universidad de Castilla-La Mancha-Iberdrola, Toledo, 29 pág.

Pfister, C., SchwarZ-Zanetti, G., Wegmann, M. y Luterbacher, J. (1998). Winter air temperature variations in western Europe during the Early and High Middle Ages (AD 750-1300). The Holocene, 8(5): 535-552.

SCHRÖTER, D. et al. (2005). Ecosystem Service Supply and Vulnerability to Global Change in Europe. Science Online.

VAlero-Garcés, B.L., Navas, A., Machín, J. y Walling, D. (1999). Sediment sources and siltation in mountain reservoirs: a case study from the Central Spanish Pyrenees. Geomorphology, 28: 23-41.

VICENTE SERRANO, S. (2004). Evolución espacio-temporal de las sequías en el sector central del valle del Ebro: causas y consecuencias ambientales. Tesis Doctoral. Universidad de Zaragoza- 1258 pp. 